\title{
Therapeutic Efficacy of Diode Laser Turbinoplasty on Nasal Obstruction Estimated with Rhinomanometry
}

\author{
Ebrahim Almulla', Dhaidan Alshammari'2, Raneem Alshaikh', Abdulrahman Almannai ${ }^{3}$, \\ Noora Althawadi', Waleed Janahi' ${ }^{4}$, Andrew Riskalla1 ${ }^{1}$, Zied Zid ${ }^{1}$ \\ ${ }^{1}$ ENT Department, King Hamad University Hospital, Muharraq, Kingdom of Bahrain \\ ${ }^{2}$ Ministry of Health, Dammam, Saudi Arabia \\ ${ }^{3}$ Ministry of Health, Manama, Kingdom of Bahrain \\ ${ }^{4}$ Head and Neck Surgery Department, University College London Hospital, London, UK \\ Email: dr.ebrahimalmulla.ent@gmail.com,dr.ebrahimalmulla.ent@gmail.com,dr.r.alshaikh@hotmail.com, \\ Abdulrahman.almannai@gmail.com, altn011@gmail.com, waleed.janahi@khuh.org.bh, Andrew.riskalla@khuh.org.bh, \\ zied.zid@khuh.org.bh
}

How to cite this paper: Almulla, E., Alshammari, D., Alshaikh, R., Almannai, A., Althawadi, N., Janahi, W., Riskalla, A. and Zid, Z. (2021) Therapeutic Efficacy of Diode Laser Turbinoplasty on Nasal Obstruction Estimated with Rhinomanometry. International Journal of Otolaryngology and Head \& Neck Surgery, 10, 487-496. https://doi.org/10.4236/ijohns.2021.106043

Received: September 7, 2021

Accepted: October 11, 2021

Published: October 14, 2021

Copyright $\odot 2021$ by author(s) and Scientific Research Publishing Inc. This work is licensed under the Creative Commons Attribution International License (CC BY 4.0).

http://creativecommons.org/licenses/by/4.0/ (c) (i) Open Access

\begin{abstract}
Objective: Diode-assisted laser turbinoplasty is a popular surgical technique that improves airflow during nasal obstruction. In this study, we aimed to evaluate the efficacy of a diode laser for turbinate hypertrophy by using rhinomanometry. Methods: This cross-sectional study included 199 patients for 13 months. Preoperative, intraoperative, and 6-week postoperative rhinomanometry values were measured. Results: The most common intraoperative symptoms were olfactory annoyance (barbecue smell) (76.4\%) and a painful burning or stinging sensation (64.3\%). The inspiratory and expiratory mean nasal airflow values increased both intraoperatively and postoperatively, illustrating the effect of decongestants and inferior turbinate surgery. Intraoperatively, inspiration improved by $262.73 \pm 196.09$ ( $\mathrm{p}<0.01$ ) and expiration by $247.94 \pm 180.05(\mathrm{p}<0.01)$. Postoperative inspiration improved by $254.03 \pm$ $199.08(\mathrm{p}<0.01)$ and expiration by $244.05 \pm 194.57(\mathrm{p}<0.01)$. Postoperative snoring $(22.6 \%, \mathrm{p}=0.026)$ and nasal obstruction $(20.2 \%, \mathrm{p}=0.042)$ were significantly higher in female than in male patients. Conclusions: The therapeutic efficacy of using diode lasers in inferior turbinate resection was established in this study on the basis of rhinomanometric data with a follow-up of 6 weeks. Postoperative nasal obstruction was effectively decreased and the other symptoms displayed a female preponderance.
\end{abstract}

\section{Keywords}

Diode-Assisted Laser Turbinoplasty, Nasal Obstruction, Turbinate 
Hypertrophy, Rhinomanometry

\section{Introduction}

Diode-assisted laser turbinoplasty is a widely used surgical method for improving airflow as a treatment for nasal obstruction [1]. Predominantly caused by hypertrophy of the inferior nasal turbinates, nasal obstruction remains one of the most common conditions encountered in rhinology practice. It can be due to mucosal thickening when the submucosal sinusoids dilation occurs, bony hypertrophy or both [2]. Swelling of the mucosa and inferior turbinates, hypertrophy of the nasal turbinates, and inflammation of the submucosal tissue as a result of collagen deposition are the cardinal mechanisms of nasal obstruction [3] [4]. Chronic obstruction with inferior turbinate hypertrophy has been observed in allergic or vasomotor rhinitis and other clinical conditions, leading to difficulty breathing, snoring, and decreased sleep quality [5]. Conservative management encompasses antihistamines, systemic steroids, and hypo-sensitization with specific immunotherapy and the restrictive use of nasal drops [6].

In some patients with bony abnormalities or refractory conditions, topical steroids are ineffective for mucosal congestion [7]. Surgical management of symptomatic hypertrophy of the nasal turbinates includes turbinectomy, turbinoplasty, and laser-assisted turbinoplasty. These methods are, however, associated with serious adverse complications such as postoperative bleeding, foul odor, hyposmia, pain, crusting, and synechiae [8] [9]. Partial or total excision of the turbinates is therefore not recommended in clinical practice [10]. Novel alternative modalities encompass $\mathrm{CO}_{2}$ laser, radiofrequency, electrocautery, and diode laser, which offer marked relief from symptoms and improve the quality of life in patients affected with inferior turbinate hypertrophy [10]. Among these methods, microdebrider-assisted inferior turbinoplasty and radiofrequency ablation have been widely investigated [11]. Recently, diode laser has attained significant popularity in terms of affordability and portability, also in having more tissue penetration and thus effectiveness in reducing parasympathetic activity will increase [12]. Diode laser treatment requires only local anesthetics and elicits controlled coagulation and ablation of soft tissue with homogenous distribution of the tissue at the surgical site [4]. The efficacy of diode laser-assisted turbinoplasty is evaluated by using rhinomanometry with topical decongestion [13]. The diode laser offers extended relief of symptoms and has a high patient acceptance rate.

Nasal airflow is measured by using peak nasal inspiratory flow rhinomanometry and acoustic rhinometry [14]. Rhinomanometry is a reliable method used to measure nasal airflow, nasal airway resistance, and transnasal pressure (the pressure difference between the nasopharynx and exterior of the nose) [14]. In recent years, there has been a marked increase in the incidence of nasal obstruc- 
tion caused by inferior turbinate hypertrophy in the younger population that is refractory to medical treatment. This scenario has led to an increase in the need for surgical intervention. In this context, we aimed in the present study to evaluate the efficacy of diode lasers for turbinate hypertrophy by using rhinomanometry.

\section{Materials and Methods}

\subsection{Subjects}

This cross-sectional study with a diagnostic study design involved 199 patients with nasal obstruction resulting from turbinate hypertrophy. It spanned a period of 13 months at the ENT outpatient clinic in King Hamad University Hospital. The history and clinical assessment of the patients was done by video endoscopy, and computed tomography scans of the sinuses were requested to rule out the presence of concurrent medical conditions (e.g., sinusitis).

Patients were included in the study if they had a history of nasal obstruction of $\geq 1$ year with constant bilateral nasal obstruction; a confirmed diagnosis of allergic rhinitis, vasomotor rhinitis, or rhinitis medicamentosa; and one or more previous conservative treatments that were unsuccessful (e.g., nasal steroid spray). Patients were excluded from the study if they had a coexisting acute infection, nasal polyps, or atrophic rhinitis (crusted, pale mucosa without hyperplastic inferior turbinates), or if they had chronic sinusitis leading to chronic nasal obstruction. Also excluded were patients who had undergone previous surgery or who were undergoing simultaneous surgeries, e.g., laser uvuloplasty, as well as those who had obstructive septal deviation requiring corrective surgery. Postoperatively, those who underwent out-fracturing/lateralization of the turbinates during surgery were also excluded.

After detailing the treatment and evaluation plan to the patients, we enrolled them in the study with their full written consent. Background information was collected according to a standard form. The proposal was approved by the Institutional Review Board and conducted ethically in accordance with the Declaration of Helsinki.

\subsection{Intervention}

Preoperative baseline rhinomanometry was measured under standardized conditions at a pressure of $150 \mathrm{~Pa}$ (T0). To avoid confounding bias, we divided the subjects into two groups by allocating them to either of the two principal surgeons performing the procedure (100 patients each). The patients were all advised to discontinue using topical nasal therapies for the week before surgery and to complete the postoperative questionnaire, which involved a subjective rating of symptoms related to nasal obstruction and complications: postoperative pain, bleeding, and infection.

Cotton pledgets containing xylocaine and $0.05 \%$ xylometazoline were inserted into each nostril twice with a 5-minute interval between applications in order to 
reduce the volume of the submucosal layer. Rhinomanometry was repeated after decongestion (T1) and the difference calculated (T1 - T0). The differences between the baseline values and those after decongestion constituted the spread of decongestion, i.e., the current degree of nasal mucosal swelling. Larger differences imply stronger improvements in nasal airflow as a result of decongestion.

Using aseptic precautions and with the patient under local anesthesia, we performed three to four laser light applications in "contact mode," drawing the fiber from the anterior to the posterior of the inferior turbinate or vice versa, according to the surgeon's training and expertise. The output power of the diode laser (wavelength, $\lambda=980 \mathrm{~mm}$ ) was preset at a standardized value of $20 \mathrm{~N}$ in continuous wave mode. The total energy applied to each inferior turbinate was $100 \mathrm{~J}$. Out-fracturing/lateralization of the turbinate was not performed. We then filled the patient's nasal cavity with antibiotic ointment and prescribed the use of nasal irrigation with saline for 4 weeks following surgery. Nasal ointment and decongestant were also given for 1 week postoperatively.

Rhinomanometry was repeated 6 weeks after the surgical procedure (T2), and the results were compared with the preoperative and intraoperative readings. The subjective questionnaire entailed a closed format with yes/no questions for all possible complications such as snoring, need for decongestants, need for nasal sprays, postoperative pain, bleeding, purulent nasal discharge, and crusting. For nasal obstruction, patients had to choose between four scored answers: deterioration ( -1$)$, no change (0), temporary improvement (1), and constant improvement (2), weighted for quantitative analysis and correlations.

\subsection{Data Analysis}

Statistical analysis was performed by using SPSS (version 25.0). An analysis of variance test was applied to observe the mean difference during inspiration and during expiration at three time periods (preoperative: $\mathrm{T} 0$, intraoperative: $\mathrm{T} 1$, and postoperative: T2). A post hoc analysis and paired sample t-test were used to compare the mean results of rhinomanometry before and after the procedure. The two-sided significance level was set to $\mathrm{p}<0.05$, with $95 \%$ confidence intervals reported and rated as signifying a clinically meaningful difference. The results obtained from rhinomanometry were then correlated with the subjective symptom improvement questionnaire. A receiver operator curve was used to obtain the sensitivity and specificity of rhinomanometry.

\section{Results}

After preoperative evaluation and application of the exclusion criteria, we performed the procedure on 199 patients, of whom $57.8 \%$ were males and $42.2 \%$ were females. A male preponderance was observed and the majority of patients were between 26 and 35 years old (35.6\%).

The major intraoperative symptoms were olfactory annoyance (barbecue smell) (76.4\%), followed by a painful burning or stinging sensation (64.3\%). A few par- 
ticipants reported sneezing or coughing (27.1\%).

The mean rhinomanometry parameters during both inspiration and expiration were recorded during three time periods: preoperative (T0), intraoperative (T1), and postoperative (T2). There was a significant increase in inspiratory ( $\mathrm{p}<$ $0.01)$ and expiratory $(\mathrm{p}<0.01)$ mean values of nasal airflow both intraoperatively and postoperatively, showing the effect of decongestants and inferior turbinate surgery. The results are shown in Table 1 . Intraoperative and postoperative time points improved significantly for both inspiration and expiration. The intraoperative values indicate the absolute spread of decongestion, which was the difference in nasal airflow measured before and after decongestion, depending on the thickness of the submucosa and the effect of decongestion (T1 - T0). Intraoperative inspiration improved by $262.73 \pm 196.09(\mathrm{p}<0.01)$ and expiration by $247.94 \pm 180.05(\mathrm{p}<0.01)$. Postoperative inspiration improved by $254.03 \pm$ 199.08 ( $\mathrm{p}<0.01)$ and expiration by $244.05 \pm 194.57(\mathrm{p}<0.01)$. There was no significant difference in inspiration or expiration or between postoperative and intraoperative values, indicating that the patients were stable. The data are shown in Table 2.

The association between gender and intraoperative and postoperative improvements are shown in Table 3. There was no significant association between intraoperative and postoperative improvements and gender. Similarly, results of the

Table 1. Rhinomanometry parameters during inspiration and expiration at various time periods (T0, T1, T3).

\begin{tabular}{ccccc}
\hline Nasal flow & Period & Mean \pm SD & F & p-value \\
\hline Inspiration (units) & T0 & $651.83 \pm 272.33$ & & \\
& T1 & $914.56 \pm 212.75$ & 86.27 & $<0.01^{\star}$ \\
& T2 & $905.86 \pm 186.26$ & & \\
Expiration (units) & T0 & $659.86 \pm 282.66$ & & \\
& T1 & $907.80 \pm 216.60$ & 73.35 & $<0.01^{*}$ \\
& T2 & $903.91 \pm 193.33$ & & \\
\hline
\end{tabular}

Table 2. Mean differences in inspiration and expiration with rhinomanometry between two different time periods.

\begin{tabular}{cccc}
\hline Nasal flow & Period & Mean difference \pm SD & p-value \\
\hline \multirow{2}{*}{ Inspiration (units) } & T1 - T0 & $262.73 \pm 196.09$ & $<0.01^{*}$ \\
& T2 - T0 & $254.03 \pm 199.08$ & $<0.01^{\star}$ \\
& T2 - T1 & $-8.69 \pm 170.97$ & 0.922 \\
\hline \multirow{2}{*}{ Expiration (units) } & T1 - T0 & $247.94 \pm 180.05$ & $<0.01^{*}$ \\
& T2 - T0 & $244.05 \pm 194.57$ & $<0.01^{*}$ \\
& T2 - T1 & $-3.89 \pm 140.72$ & 0.985 \\
\hline
\end{tabular}

${ }^{*} \mathrm{p}<0.05$ was considered statistically significant. 
Table 3. Association between gender and intraoperative and postoperative improvements.

\begin{tabular}{ccccc}
\hline \multirow{2}{*}{ Nasal flow } & Period & \multicolumn{2}{c}{ Mean difference \pm SD } & Mann-Whitney \\
\cline { 3 - 4 } & & Male & Female & test p-value \\
\hline \multirow{2}{*}{ Inspiration (units) } & T1 - T0 & $274.92 \pm 205.51$ & $246.04 \pm 182.30$ & 0.30 \\
& T2 - T0 & $251.77 \pm 204.07$ & $257.13 \pm 193.22$ & 0.90 \\
\hline \multirow{2}{*}{ Expiration (units) } & T1 - T0 & $250.49 \pm 188.58$ & $244.45 \pm 168.74$ & 0.85 \\
& T2 - T0 & $244.20 \pm 189.70$ & $243.84 \pm 202.19$ & 0.70 \\
\hline
\end{tabular}

$\mathrm{p}<0.05$ was considered statistically significant.

Kruskal-Wallis test showed that the impact of different age groups was not significant, implying that the improvements were not age dependent (results not shown).

Postoperative symptoms among the study subjects are shown in Table 4. None of the patients required nasal packing and no complications such as bleeding occurred immediately after surgical intervention. Postoperatively, male patients showed better outcomes than female patients did, as snoring $(22.6 \%, \mathrm{p}=0.026)$ and nasal obstruction $(20.2 \%, p=0.042)$ were more common in females. The need for a decongestant was higher in females than in males but was not significant $(19.0 \%$ vs $10.4 \%$, respectively; $\mathrm{p}=0.06)$. Furthermore, purulent nasal discharge and crusting, although more commonly reported in women than in men, was nonsignificant.

The efficacy of diode lasers in inferior turbinate resection in reducing symptoms pre- and postoperatively are shown in Table 5 . Significant improvements were seen in all symptoms after surgical intervention. Nasal obstruction $(14.6 \%$ vs $58.3 \%$; $\mathrm{p}<0.001$ ), snoring ( $16.1 \%$ vs $41.7 \%$; $\mathrm{p}=0.001$ ), need for nasal spray (24.1\% vs $97 \%$; $\mathrm{p}<0.001)$, and need for nasal decongestant $(14.1 \%$ vs $84.9 \%$; $<<$ 0.001 ) were significantly reduced after surgery compared with before surgery.

\section{Discussion}

Diode laser-assisted turbinate surgery is a popular procedure for improving nasal airflow. It is better absorbed by tissue than that of Nd: YAG lasers but with tissue ablation effects similar to a carbon dioxide laser [1]. Volk et al. [4] evaluated diode laser-assisted nasal airflow and reported that rhinomanometry with decongestion has a predictive value for the enhancement of airflow after surgery.

This study is unique in using rhinomanometry as the sole measurement tool to assess the diode laser efficacy through analysis of symptoms and postoperative morbidities. In a previous study by Cakli et al. [13], they reported the outcomes of diode laser turbinoplasty by using acoustic rhinometry and showed satisfactory success rates for diode laser $(\mathrm{k}=980 \mathrm{~nm})$-assisted turbinate surgery, both objectively and subjectively.

In our study, male preponderance was observed, which is in line with the results of studies by Kankaanpää et al. [2], Cakli et al. [13], and Veit et al. [15]. In 
Table 4. Distribution of postoperative symptoms by gender.

\begin{tabular}{|c|c|c|}
\hline Symptom & Frequency, n (\%) & p-value \\
\hline \multicolumn{3}{|l|}{ Nasal obstruction } \\
\hline Male & $12(10.4)$ & $0.042^{*}$ \\
\hline Female & $17(20.2)$ & \\
\hline \multicolumn{3}{|l|}{ Snoring } \\
\hline Male & $13(11.3)$ & $0.026^{*}$ \\
\hline Female & $19(22.6)$ & \\
\hline \multicolumn{3}{|l|}{ Need for decongestant } \\
\hline Male & $12(10.4)$ & 0.065 \\
\hline Female & $16(19.0)$ & \\
\hline \multicolumn{3}{|l|}{ Need for spray } \\
\hline Male & $25(21.7)$ & 0.226 \\
\hline Female & $23(27.4)$ & \\
\hline \multicolumn{3}{|l|}{ Bleeding from nose } \\
\hline Male & $2(2.4)$ & 0.56 \\
\hline Female & $2(2.4)$ & \\
\hline \multicolumn{3}{|l|}{ Purulent nasal discharge } \\
\hline Male & $0(0.0)$ & 0.07 \\
\hline Female & $3(3.6)$ & \\
\hline \multicolumn{3}{|l|}{ Crusting } \\
\hline Male & $34(29.6)$ & 0.10 \\
\hline Female & $33(39.3)$ & \\
\hline
\end{tabular}

${ }^{*} \mathrm{p}<0.05$ was considered statistically significant.

Table 5. Effect of diode lasers in the reduction of symptoms.

\begin{tabular}{cccc}
\hline Symptom & Preoperative, $\mathbf{n}(\%)$ & Postoperative, $\mathrm{n}(\%)$ & p-value \\
\hline Nasal obstruction & $116(58.3)$ & $29(14.6)$ & $<0.001^{\star}$ \\
Snoring & $83(41.7)$ & $32(16.1)$ & $0.001^{\star}$ \\
Need for nasal spray & $193(97.0)$ & $48(24.1)$ & $<0.001^{\star}$ \\
Need for nasal decongestant & $169(84.9)$ & $28(14.1)$ & $<0.001^{\star}$ \\
\hline
\end{tabular}

${ }^{\star} \mathrm{p}<0.05$ was considered statistically significant.

our study, the majority of the patients were between 26 and 35 years old, similar to the results reported by Kankaanpää et al. [2]. Nasal obstruction, snoring, and the need for nasal decongestants and sprays were the predominant preoperative symptoms observed in our study. Preoperative nasal findings for obstruction $(116,58.3 \%)$ and snoring $(83,41.7 \%)$ were in agreement with the values reported 
in the literature and corroborated findings from similar studies [3] [16] [17] [18].

Objective analysis revealed significant improvements in inspiratory and expiratory values at intraoperative and postoperative time points from the initial rhinomanometric values. Only $14.6 \%$ of patients had nasal obstruction 6 weeks postoperatively, indicating improved airflow in more than $75 \%$ of patients, similar to that reported in the study by Volk et al. [4]. A similar significant increase in postoperative inspiratory and expiratory ventilation was also shown as that for the absolute spread of decongestion, i.e., the difference in nasal airflow before and after decongestion. In a prospective study, Parida et al. [17] demonstrated significant improvements in visual analog scale scores with diode laser turbinate reduction at the end of 1 week, 1 month, 3 months, and 6 months.

The findings of our study indicate that neither age nor gender had any influence on the intraoperative and postoperative mean differences. The subjective analysis of symptoms postoperatively differed according to gender, however, showing a significant prevalence of nasal obstruction and snoring in women. Although the remaining postoperative symptoms were also more common in women than in men, they were not significant, raising questions about the reason for the more frequent occurrence.

Caffier et al. [16] established endonasal outpatient diode laser turbinoplasty as an effective, safe, and well-tolerated procedure in treating therapy-resistant inferior turbinate hypertrophy. Our findings were also significant regarding improvements in postoperative symptoms at 6 weeks after surgery relative to their prior states, confirming the efficacy of diode laser treatment in inferior turbinate resection.

\section{Limitations}

Because this was a single-center study, the results may not represent the situation in other healthcare institutions across the country. The limitations of convenience sampling are also applicable here, including the inability to generalize the results of the survey to the population as a whole and under- or over-representation of the population. Moreover, a delay in data collection was unpreventable. Rhinometry required maintenance, which took approximately 3 months. Furthermore, the COVID-19 pandemic further reduced the flow of patients because of the implementation of teleconsultation in the hospital.

\section{Conclusion}

From the rhinomanometric findings, we affirmed the therapeutic efficacy of using diode lasers in inferior turbinate resection in this clinical study with a follow-up of 6 weeks. Postoperative nasal obstruction was substantially decreased in both male and female patients, but that and other symptoms were more common in female than in male patients. The age and gender of the patients did not influence the rhinomanometric findings. 


\section{Recommendations}

An interesting result of this study is the propensity in female patients versus male patients to have postoperative symptoms, although there was a male preponderance. Studies to confirm such a finding, as well as a survey to assess the reasons for such an effect, could be considered in the future.

Given the paucity of research using rhinomanometry, further studies could also be considered with longer follow-up periods to assess long-term outcomes and explore mucociliary clearance mechanisms. The present study could also be replicated on a larger sample to generalize the findings and extend them to other institutions.

\section{Ethical Committee Approval}

The study was approved by the Institutional Review Board at King Hamad University Hospital, Bahrain (Approval Date: March 4, 2018, Approval Number: 203/2018).

\section{Financial Disclosure}

The authors declared that this study received no financial support.

\section{Informed Consent}

Informed consent was obtained from all patients who met the inclusion criteria.

\section{Conflicts of Interest}

The authors have no conflicts of interest to declare.

\section{References}

[1] Ravichandran, S., Ramasamy, K., Parida, P., et al. (2020) Comparison of Efficacy of Potassium Titanyl Phosphate Laser \& Diode Laser in the Management of Inferior Turbinate Hypertrophy: A Randomized Controlled Trial. The Indian Journal of Medical Research, 151, 578-584. https://doi.org/10.4103/ijmr.IJMR 42418

[2] Kankaanpää, A., Harju, T. and Numminen, J. (2020) The Effect of Inferior Turbinate Surgery on Quality of Life: A Randomized, Placebo-Controlled Study. Ear, Nose, and Throat Journal. https://doi.org/10.1177/0145561320927944

[3] Kassab, A.N., Rifaat, M. and Madian, Y. (2012) Comparative Study of Management of Inferior Turbinate Hypertrophy Using Turbinoplasty Assisted by Microdebrider or $980 \mathrm{~nm}$ Diode Laser. The Journal of Laryngology and Otology, 126, 1231-1237. https://doi.org/10.1017/S0022215112002320

[4] Volk, G.F., Pantel, M., Guntinas-Lichius, O. and Wittekindt, C. (2010) Prognostic Value of Anterior Rhinomanometry in Diode Laser Turbinoplasty. Archives of Otolaryngology_Head and Neck Surgery, 136, 1015-1019. https://doi.org/10.1001/archoto.2010.161

[5] Ye, T. and Zhou, B. (2015) Update on Surgical Management of Adult Inferior Turbinate Hypertrophy. Current Opinion in Otolaryngology and Head and Neck Surgery, 23, 29-33. https://doi.org/10.1097/MOO.0000000000000130

[6] Mehuys, E., Gevaert, P., Brusselle, G., et al. (2014) Self-Medication in Persistent Rhi- 
nitis: Overuse of Decongestants in Half of the Patients. The Journal of Allergy and Clinical Immunology: In Practice, 2, 313-319. https://doi.org/10.1016/j.jaip.2014.01.009

[7] Teichgraeber, J.F., Gruber, R.P. and Tanna, N. (2016) Surgical Management of Nasal Airway Obstruction. Clinics in Plastic Surgery, 43, 41-46. https://doi.org/10.1016/j.cps.2015.09.006

[8] Chhabra, N. and Houser, S.M. (2012) Surgical Options for the Allergic Rhinitis Patient. Current Opinion in Otolaryngology and Head and Neck Surgery, 20, 199-204. https://doi.org/10.1097/MOO.0b013e328352b821

[9] Halderman, A. and Sindwani, R. (2015) Surgical Management of Vasomotor Rhinitis: A Systematic Review. American Journal of Rhinology and Allergy, 29, 128-134. https://doi.org/10.2500/ajra.2015.29.4141

[10] Prokopakis, E.P., Koudounarakis, E.I. and Velegrakis, G.A. (2014) Efficacy of Inferior Turbinoplasty with the Use of $\mathrm{CO}(2)$ Laser, Radiofrequency, and Electrocautery. American Journal of Rhinology and Allergy, 28, 269-272. https://doi.org/10.2500/ajra.2014.28.4044

[11] Bhandarkar, N.D. and Smith, T.L. (2010) Outcomes of Surgery for Inferior Turbinate Hypertrophy. Current Opinion in Otolaryngology and Head and Neck Surgery, 18, 49-53. https://doi.org/10.1097/MOO.0b013e328334d974

[12] Doreyawar, V., Gadag, R.P., Manjunath, et al. (2018) Inferior Turbinate Reduction: Diode Laser or Conventional Partial Turbinectomy? Ear, Nose, and Throat Journal, 97, E15-E19.

[13] Cakli, H., Cingi, C., Güven, E., Gurbuz, M.K. and Kaya, E. (2012) Diode Laser Treatment of Hypertrophic Inferior Turbinates and Evaluation of the Results with Acoustic Rhinometry. European Archives of Oto-Rhino-Laryngology, 269, 2511-2517. https://doi.org/10.1007/s00405-012-1963-1

[14] Chaaban, M. and Corey, J.P. (2011) Assessing Nasal Air Flow: Options and Utility. Proceedings of the American Thoracic Society, 8, 70-78. https://doi.org/10.1513/pats.201005-034RN

[15] Veit, J.A., Nordmann, M., Dietz, B., et al. (2017) Three Different Turbinoplasty Techniques Combined with Septoplasty: Prospective Randomized Trial. Laryngoscope, 127, 303-308. https://doi.org/10.1002/lary.26264

[16] Caffier, P.P., Scherer, H., Neumann, K., et al. (2011) Diode Laser Treatment in Therapy-Resistant Allergic Rhinitis: Impact on Nasal Obstruction and Associated Symptoms. Lasers in Medical Science, 26, 57-67. https://doi.org/10.1007/s10103-010-0813-X

[17] Parida, P.K., Surianarayanan, G., Alexander, A., Saxena, S.K. and Santhosh, K. (2013) Diode Laser Turbinate Reduction in the Treatment of Symptomatic Inferior Turbinate Hypertrophy. Indian Journal of Otolaryngology and Head and Neck Surgery, 65, 350-355. https://doi.org/10.1007/s12070-012-0515-8

[18] Gupta, P., Kc, T. and Regmi, D. (2018) Diode Laser Turbinate Reduction in Allergic Rhinitis: A Cross-Sectional Study. Journal of the Nepal Medical Association, 56, 949 952. https://doi.org/10.31729/jnma.3870 